\title{
A variety of explicit exact and soliton type solutions of conformable fractional Equal-Width equations
}

\author{
Asim Zafar ${ }^{1}$, Alper Korkmaz ${ }^{2, *}$, Hadi Rezazadeh ${ }^{3}$ \\ ${ }^{1}$ Department of Mathematics, COMSATS University Islamabad, \\ Vehari Campus, Pakistan. \\ ${ }^{2}$ Department of Mathematics, ÇAKU, Çankırı, Turkey. \\ ${ }^{3}$ Faculty of Engineering Technology, Amol University of Special Modern Technologies, \\ Amol, Iran.
}

\begin{abstract}
Exact and soliton type solutions have great importance in propagation of surface waves, fluid dynamics, optics, and many other fields of nonlinear sciences. In this study, the explicit and exact soliton type solutions for two space-time fractional EqualWidth (FEW) equations with conformable derivative are procured via the hyperbolic function approach. The wave type solutions are represented in some hyperbolic and trigonometric functions.
\end{abstract}

Keywords: Space-time fractional Equal-Width equations; Conformable derivative; Hyperbolic function approach; Exact soliton solutions.

\section{Introduction}

A very long while back, more general types of differential equations were represented as fractional differential equations. Different phenomena in numerous natural, physical and social science fields are demonstrated by those equations [1]. The renderings of broadcasting, diffusive convection, Fokker- Plank type, growth, and other differential equations are extended by utilizing fractional derivatives [2]. The study of fractional calculus and the fractional differential equations has obtained great interest for researchers in the field of nonlinear sciences [3-5]. Many definitions of fractional derivatives, Like Hilfer, RiemannLiouville, Caputo form and so on, have been introduced in the literature [6-8], but the most

\footnotetext{
*Corresponding author: alperkorkmaz7@gmail.com
} 
fascinating definition and the geometrical explanation for complex fractional transform and fractional derivative are given in [9-11].

The regularized long wave (RLW) equation is an alternative description of non-linear dispersive waves to the more usual Korteweg-de Vries (KdV) equation. It has solitary wave solutions of a rather general type $[12,13]$. A less well-known alternative, suggested by Morrison et al. [14] to use as a model PDE for the simulation of one-dimensional wave propagation in nonlinear media with dispersion processes, is called equal width equation (EWE) which also has solitary wave solutions, but of a less general type. Due to having analytical solutions, the EWE also attracts many researchers studying numerical techniques for nonlinear initial boundary value problems defined with PDEs [15-18]. Various analytical methods have been presented in the literature to explore exact solutions to nonlinear PDEs [19-24]. Furthermore, some other excellent works like the expansion method based on Sine-Gordon equation [25], the method of projective Riccati [26], the generalized Kudryashov method $[27,28]$, a modified form of Kudryashov and functional variable methods [29-33] have been done by different researchers. In [34-36], the auxiliary equation, the

extended tanh-function, the improved $\tan \left(\frac{\phi(\eta)}{2}\right)$-expansion methods and the exp function approach have been explored for discrete and fractional order PDEs as well. In particular, Ali and Hassan, Hosseini et al., Zayed and Al-Nowehy all have utilized the $\exp _{a}$ function method in [37-40] respectively. Furthermore, the hyperbolic function approach has been explored in $[39,41,42]$ to procure exact solutions of nonlinear PDEs.

This paper aims to explore the hyperbolic function approach to generate new explicit soliton type solutions for the space-time FEW equations $[13,14,43]$ defined with conformable derivative and using the compatible traveling wave transformation. The organization of this paper is as follows: Section 2 presents a brief description of the conformable derivative and the hyperbolic function approach. Section 3 illustrate how to utilize the hyperbolic function approach for producing new explicit exact solutions for space-time FEW equations. The latter part summarizes results of the current study.

\section{Conformable fractional derivatives and the method of so- lutions}

Recall the definition of the conformable fractional derivative with some of its properties. Definition 1 Suppose $h: \mathbb{R}^{+} \rightarrow \mathbb{R}$ be a function. Then, for all $t>0$,

$$
D_{t}^{\gamma}(h(t))=\lim _{\varepsilon \rightarrow 0} \frac{h\left(t+\varepsilon t^{1-\gamma}\right)-h(t)}{\varepsilon}
$$

is known as the conformable fractional derivative of $h$ of order $\gamma, 0<\gamma \leq 1$. Some useful properties are being listed as follows:

$D_{t}^{\gamma}(a h+b g)=a D_{t}^{\gamma}(h)+b D_{t}^{\gamma}(g)$, for all $a, b \in \mathbb{R}$

$D_{t}^{\gamma}(h g)=h D_{t}^{\gamma}(g)+g D_{t}^{\gamma}(h)$ 
Let $h: \mathbb{R}^{+} \rightarrow \mathbb{R}$ be a differentiable and $\gamma$-differentiable function, $g$ be a differentiable function defined in the range of $h$.

$$
D_{t}^{\gamma}(h \circ g(t))=t^{1-\gamma} g^{\prime}(t) h^{\prime}(g(t)) .
$$

On the top of that, the following rules hold.

$D_{t}^{\gamma}\left(t^{p}\right)=p t^{p-\gamma}$, for all $p \in \mathbb{R}$

$D_{t}^{\gamma}(\lambda)=0$, where $\lambda$ is constant.

$D_{t}^{\gamma}(h / g)=\frac{g D_{t}^{\gamma}(h)-h D_{t}^{\gamma}(g)}{g^{2}}$.

Conjointly, if $h$ is differentiable, then $D_{t}^{\gamma}(h(t))=t^{1-\gamma} \frac{d h(t)}{d t}$.

\subsection{A transitory explanation of hyperbolic function method}

The present subsection provides a brief explanation for hyperbolic function method $[41,42]$ in engendering new exact solutions to nonlinear conformable space-time FEW and modified FEW equations. For this purpose, suppose that a nonlinear conformable space-time FDE that can be presented in the form

$$
F\left(u, D_{t}^{\gamma} u, D_{x}^{\beta} u, D_{t t}^{2 \gamma} u, D_{x x}^{2 \beta} u, \ldots\right)=0
$$

The FDE defined in Eq.(1) can be changed into the following nonlinear ODE of integer order

$$
P\left(U, U^{\prime}, U^{\prime \prime}, \ldots,\right)=0
$$

with the use of following transformation

$$
u(x, t)=U(\xi), \xi=k \frac{x^{\beta}}{\beta}-l \frac{t^{\gamma}}{\gamma},
$$

where $k, l$ are nonzero arbitrary constants.

Let us try to search a non-trivial solution to the Eq.(2) in the following form

$$
U(\xi)=A_{0}+\sum_{i=1}^{N} \sinh ^{i-1}(\omega)\left[B_{i} \sinh (\omega)+A_{i} \cosh (\omega)\right]
$$

where $w$ is some specific functions. By calculating the positive integer $N$, setting the Eq.(4) in Eq.(2), and comparing the coefficients, we will find a set of nonlinear algebraic equations whose solution, finally provides explicit exact solutions of the Eq.(1). It is worth mentioning that using the separation of variables techniques from $\frac{d \omega}{d \xi}=\sinh (\omega)$ we find $\sinh (\omega)=-\csc (\xi)$ and $\cosh (\omega)=-\operatorname{coth}(\xi)$. Likewise, it is obvious that from $\frac{d w}{d \xi}=\cosh (\omega)$, we find $\sinh (\omega)=-\cot (\xi)$ and $\cosh (\omega)=\csc (\xi)$. Accordingly, the solution (4) can be rewritten as

$$
U(\xi)=A_{0}+\sum_{i=1}^{N}\left(-\operatorname{csch}^{i-1}(\xi)\right)\left[-B_{i} \operatorname{csch}(\xi)-A_{i} \operatorname{coth}(\xi)\right],
$$


and

$$
U(\xi)=A_{0}+\sum_{i=1}^{N}\left(-\cot ^{i-1}\right)(\xi)\left[-B_{i} \cot (\xi)+A_{i} \csc (\xi)\right]
$$

\section{Explicit exact solutions for space-time fractional modified EW equation}

Consider the following space-time fractional modified EW equation [43,44], for finding its exact solutions via hyperbolic function approach.

$$
D_{t}^{\gamma} u(x, t)+\epsilon D_{x}^{\gamma} u^{3}(x, t)-\delta D_{x x t}^{3 \gamma} u(x, t)=0, \quad t>0 \quad 0<\gamma \leq 1,
$$

Taking the transformation (3) and integrating once w.r.t. $\xi$, we get

$$
\delta l k^{2} U^{\prime \prime}-l U+\epsilon k U^{3}=0 .
$$

Setting the constant of integration equals to zero and balance the terms $U^{\prime \prime}$ and $U^{3}$, gives $N=1$, the nontrivial solution 5 (a) reduces to:

$$
U(\xi)=A_{0}+A_{1} \cosh (\omega)+B_{1} \sinh (\omega)
$$

Case-1: $\frac{d \omega}{d \xi}=\sinh (\omega)$ By setting the above non-trivial solution in reduced equation Eq.(6) and equating the coefficients to zero in the resultant equation, a nonlinear algebraic set of equations is obtained which its solution yields the following new exact solutions to the conformable space-time fractional modified EW equation

$$
\begin{gathered}
\text { Case }-1.1: A_{0}=0, A_{1}=-\sqrt[4]{\frac{-\delta}{2}} \sqrt{\frac{l}{\epsilon}}, B_{1}= \pm \sqrt[4]{\frac{-\delta}{2}} \sqrt{\frac{l}{\epsilon}}, k=i \sqrt{\frac{2}{\delta}} \\
u_{1}(x, t)=\frac{(1-i) \sqrt[4]{2} \sqrt[4]{\delta} \sqrt{l}}{2 \sqrt{\epsilon}} \tanh \left(\frac{k \frac{x^{\beta}}{\beta}-l \frac{t^{\gamma}}{\gamma}}{2}\right) \\
u_{2}(x, t)=\frac{(1-i) \sqrt[4]{2} \sqrt[4]{\delta} \sqrt{l}}{2 \sqrt{\epsilon}} \operatorname{coth}\left(\frac{k \frac{x^{\beta}}{\beta}-l \frac{t^{\gamma}}{\gamma}}{2}\right) \\
A_{0}=0, A_{1}=\sqrt[4]{\frac{-\delta}{2}} \sqrt{\frac{l}{\epsilon}}, B_{1}=\mp \sqrt[4]{\frac{-\delta}{2}} \sqrt{\frac{l}{\epsilon}}, k=i \sqrt{\frac{2}{\delta}} \\
u_{3}(x, t)=\frac{(-2)^{3 / 4} \sqrt[4]{\delta} \sqrt{l}}{\sqrt{\epsilon}} \sinh ^{2}\left(\frac{k \frac{x^{\beta}}{\beta}-l \frac{t^{\gamma}}{\gamma}}{2}\right) \operatorname{csch}\left(k \frac{x^{\beta}}{\beta}-l \frac{t^{\gamma}}{\gamma}\right) \\
u_{4}(x, t)=-\frac{(1-i) \sqrt[4]{\delta} \sqrt{l}}{2^{3 / 4} \sqrt{\epsilon}}\left(\operatorname{coth}\left(k \frac{x^{\beta}}{\beta}-l \frac{t^{\gamma}}{\gamma}\right)+\operatorname{csch}\left(k \frac{x^{\beta}}{\beta}-l \frac{t^{\gamma}}{\gamma}\right)\right)
\end{gathered}
$$




$$
\begin{aligned}
& \text { Case }-1.2: A_{0}=0, A_{1}=\mp \sqrt[4]{\frac{-\delta}{2}} \sqrt{\frac{l}{\epsilon}}, B_{1}=\mp \sqrt[4]{\frac{-\delta}{2}} \sqrt{\frac{l}{\epsilon}}, \quad k=-i \sqrt{\frac{2}{\delta}} \\
& u_{5,6}(x, t)=\mp \sqrt[4]{\frac{-\delta}{2}} \sqrt{\frac{l}{\epsilon}}\left(\operatorname{coth}\left(k \frac{x^{\beta}}{\beta}-l \frac{t^{\gamma}}{\gamma}\right)+\operatorname{csch}\left(k \frac{x^{\beta}}{\beta}-l \frac{t^{\gamma}}{\gamma}\right)\right) \\
& A_{0}=0, A_{1}=\mp \sqrt[4]{\frac{-\delta}{2}} \sqrt{\frac{l}{\epsilon}}, B_{1}= \pm \sqrt[4]{\frac{-\delta}{2}} \sqrt{\frac{l}{\epsilon}}, k=-i \sqrt{\frac{2}{\delta}} \\
& u_{7,8}(x, t)=\mp \sqrt[4]{\frac{-\delta}{2}} \sqrt{\frac{l}{\epsilon}} \tanh \left(\frac{k \frac{x^{\beta}}{\beta}-l \frac{t^{\gamma}}{\gamma}}{2}\right) \\
& \text { Case }-1.3: A_{0}=0, A_{1}=0, B_{1}= \pm \frac{\sqrt{2} \sqrt[4]{\delta} \sqrt{l}}{\sqrt{\epsilon}}, k=-\frac{1}{\sqrt{\delta}} \\
& u_{9,10}(x, t)= \pm \frac{\sqrt{2} \sqrt[4]{\delta} \sqrt{l}}{\sqrt{\epsilon}} \operatorname{csch}\left(-\frac{1}{\sqrt{\delta}} \frac{x^{\gamma}}{\gamma}-l \frac{t^{\gamma}}{\gamma}\right) \\
& A_{0}=0, A_{1}=0, B_{1}= \pm \frac{i \sqrt{2} \sqrt[4]{\delta} \sqrt{l}}{\sqrt{\epsilon}}, k=\frac{1}{\sqrt{\delta}} \\
& u_{11,12}(x, t)= \pm \frac{i \sqrt{2} \sqrt[4]{\delta} \sqrt{l}}{\sqrt{\epsilon}} \operatorname{csch}\left(\frac{1}{\sqrt{\delta}} \frac{x^{\gamma}}{\gamma}-l \frac{t^{\gamma}}{\gamma}\right) \\
& \text { Case }-1.4: A_{0}=0, A_{1}=\mp \sqrt[4]{-2 \delta} \sqrt{\frac{l}{\epsilon}}, B_{1}=0, k=\frac{-i}{\sqrt{2 \delta}} \\
& u_{13,14}(x, t)=\mp \sqrt[4]{-2 \delta} \sqrt{\frac{l}{\epsilon}} \operatorname{coth}\left(k \frac{x^{\beta}}{\beta}-l \frac{t^{\gamma}}{\gamma}\right) \\
& A_{0}=0, A_{1}=\mp(-1) \sqrt[3 / 4]{4} \sqrt{2 \delta} \sqrt{\frac{l}{\epsilon}}, B_{1}=0, k=\frac{i}{\sqrt{2 \delta}} \\
& u_{15,16}(x, t)=\mp(1-i) \sqrt[4]{\frac{\delta}{2}} \sqrt{\frac{l}{\epsilon}} \operatorname{coth}\left(k \frac{x^{\beta}}{\beta}-l \frac{t^{\gamma}}{\gamma}\right)
\end{aligned}
$$


Case-2: $\frac{d \omega}{d \xi}=\cosh (\omega)$ and for $N=1$, a set of nonlinear equations is obtained and whose solution yields

$$
\begin{aligned}
& \text { Case }-2.1: A_{0}=0, \quad A_{1}=\mp i \sqrt[4]{\frac{\delta}{2}} \sqrt{\frac{l}{\epsilon}}, B_{1}=\mp i \sqrt[4]{\frac{\delta}{2}} \sqrt{\frac{l}{\epsilon}}, k=\sqrt{\frac{2}{\delta}} \\
& u_{1,2}(x, t)= \pm i \sqrt[4]{\frac{\delta}{2}} \sqrt{\frac{l}{\epsilon}} \tan \left(\frac{\sqrt{\frac{2}{\delta}} \frac{x^{\gamma}}{\gamma}-l \frac{t^{\gamma}}{\gamma}}{2}\right) \\
& A_{0}=0, \quad A_{1}=\mp i \sqrt[4]{\frac{\delta}{2}} \sqrt{\frac{l}{\epsilon}}, \quad B_{1}= \pm i \sqrt[4]{\frac{\delta}{2}} \sqrt{\frac{l}{\epsilon}}, k=\sqrt{\frac{2}{\delta}} \\
& u_{3,4}(x, t)= \pm i \sqrt[4]{\frac{\delta}{2}} \sqrt{\frac{l}{\epsilon}} \cot \left(\frac{\sqrt{\frac{2}{\delta}} \frac{x^{\gamma}}{\gamma}-l \frac{t^{\gamma}}{\gamma}}{2}\right) \\
& \text { Case }-2.2: A_{0}=0, \quad A_{1}=\mp(1+i) \sqrt[4]{\delta} \sqrt{\frac{l}{\epsilon}}, \quad B_{1}=0, \quad k=\frac{-i}{\sqrt{\delta}} \\
& u_{5,6}(x, t)= \pm(1+i) \sqrt[4]{\delta} \sqrt{\frac{l}{\epsilon}} \csc \left(\frac{-i}{\sqrt{\delta}} \frac{x^{\gamma}}{\gamma}-l \frac{t^{\gamma}}{\gamma}\right) \\
& A_{0}=0, A_{1}=\mp(1-i) \sqrt[4]{\delta} \sqrt{\frac{l}{\epsilon}}, B_{1}=0, k=\frac{i}{\sqrt{\delta}} \\
& u_{7,8}(x, t)= \pm(1-i) \sqrt[4]{\delta} \sqrt{\frac{l}{\epsilon}} \csc \left(\frac{i}{\sqrt{\delta}} \frac{x^{\gamma}}{\gamma}-l \frac{t^{\gamma}}{\gamma}\right) \\
& \text { Case }-2.3: A_{0}=0, A_{1}=0, B_{1}=\mp \sqrt[4]{2 \delta} \sqrt{\frac{l}{\epsilon}}, k=\frac{-1}{\sqrt{2 \delta}} \\
& u_{9,10}(x, t)=\mp \sqrt[4]{2 \delta} \sqrt{\frac{l}{\epsilon}} \cot \left(\frac{-1}{\sqrt{2 \delta}} \frac{x^{\gamma}}{\gamma}-l \frac{t^{\gamma}}{\gamma}\right) \\
& A_{0}=0, \quad A_{1}=0, \quad B_{1}=\mp i \sqrt[4]{2 \delta} \sqrt{\frac{l}{\epsilon}}, \quad k=\frac{1}{\sqrt{2 \delta}} \\
& u_{11,12}(x, t)=\mp i \sqrt[4]{2 \delta} \sqrt{\frac{l}{\epsilon}} \cot \left(\frac{1}{\sqrt{2 \delta}} \frac{x^{\gamma}}{\gamma}-l \frac{t^{\gamma}}{\gamma}\right)
\end{aligned}
$$




$$
\begin{aligned}
\text { Case }-2.4: A_{0} & =0, \quad A_{1}=\mp \sqrt[4]{\frac{\delta}{2}} \sqrt{\frac{l}{\epsilon}}, \quad B_{1}=\mp \sqrt[4]{\frac{\delta}{2}} \sqrt{\frac{l}{\epsilon}}, \quad k=-\sqrt{\frac{2}{\delta}} \\
u_{13,14}(x, t) & = \pm \sqrt[4]{\frac{\delta}{2}} \sqrt{\frac{l}{\epsilon}} \tan \left(\frac{k \frac{x^{\beta}}{\beta}-l \frac{t^{\gamma}}{\gamma}}{2}\right) \\
A_{0} & =0, \quad A_{1}=\mp \sqrt[4]{\frac{\delta}{2}} \sqrt{\frac{l}{\epsilon}}, B_{1}= \pm \sqrt[4]{\frac{\delta}{2}} \sqrt{\frac{l}{\epsilon}}, k=-\sqrt{\frac{2}{\delta}} \\
u_{15,16}(x, t) & = \pm \sqrt[4]{\frac{\delta}{2}} \sqrt{\frac{l}{\epsilon}}\left(\cot \left(k \frac{x^{\beta}}{\beta}-l \frac{t^{\gamma}}{\gamma}\right)+\csc \left(k \frac{x^{\beta}}{\beta}-l \frac{t^{\gamma}}{\gamma}\right)\right)
\end{aligned}
$$

\subsection{Numerical simulation for solutions of space-time fractional modified EWE}

The obtained solutions of Eq.(5) are graphed here for different $\gamma$-values corresponding to $\epsilon=1=l$, and $\delta=1$.

Case-I: The figures $1(\mathrm{a})-1(\mathrm{~d})$ and $2(\mathrm{a})-2(\mathrm{~d})$ reveal the two hyperbolic function solutions given in Eq. 8 of Eq.5 for $\gamma=0.5,1$ and $t=0$ respectively.

Case-II: The figures $3(\mathrm{a})-3(\mathrm{~b})$ and $3(\mathrm{c})-3(\mathrm{~d})$ present two periodic wave solutions given in Eq.17 of Eq.5 for $\gamma=0.5$ and $\gamma=1$ respectively.

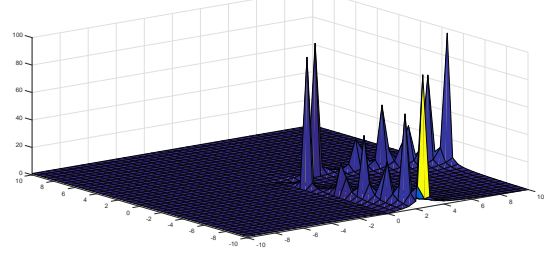

(a) $\gamma=0.5$

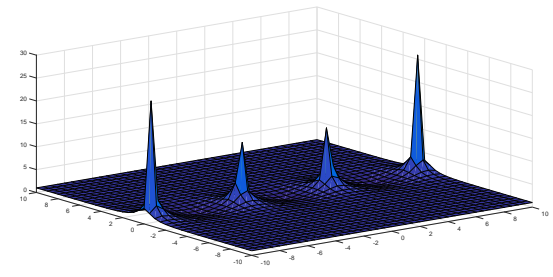

(c) $\gamma=1$

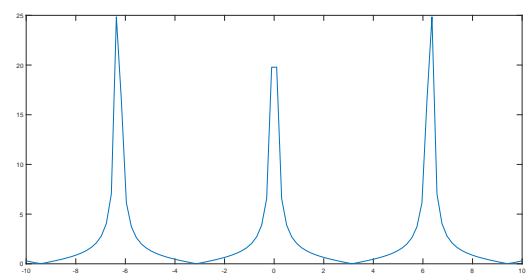

(b) $\gamma=0.5 \& t=0$

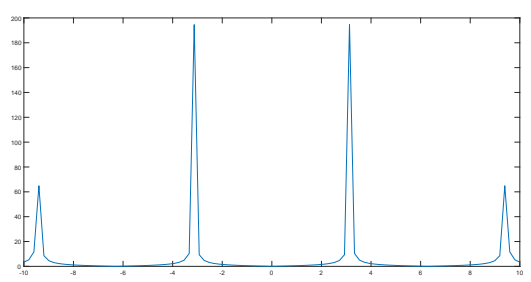

(d) $\gamma=1 \& t=0$

Figure 1: Solitary wave profile of $u_{1}$ appears in Eq.8 


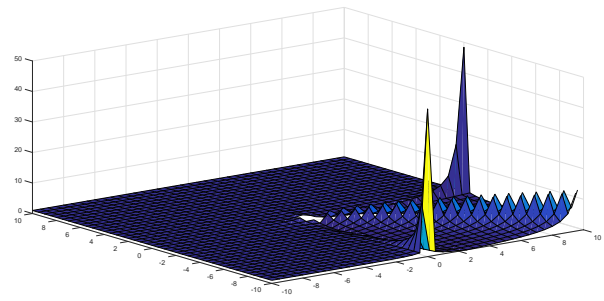

(a) $\gamma=0.5$

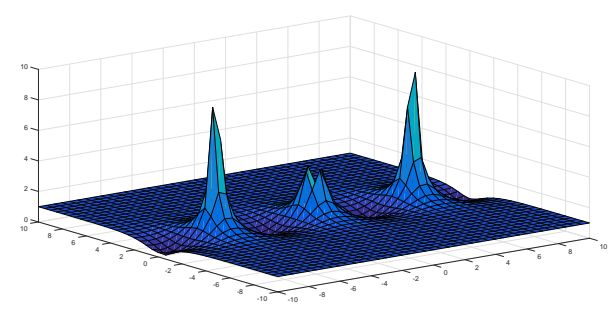

(c) $\gamma=1$

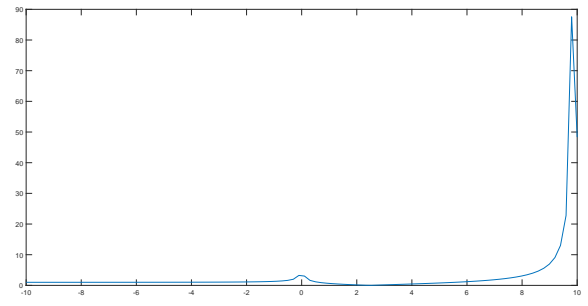

(b) $\gamma=0.5, t=0$

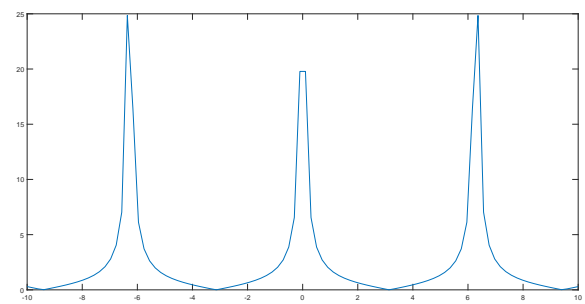

(d) $\gamma=1, \& t=0$

Figure 2: Solitary wave profile of $u_{2}$ appears in Eq.8

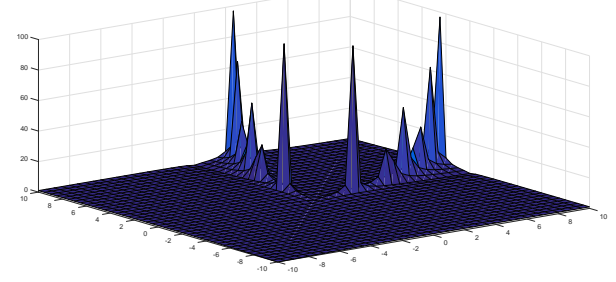

(a) $\gamma=0.5$

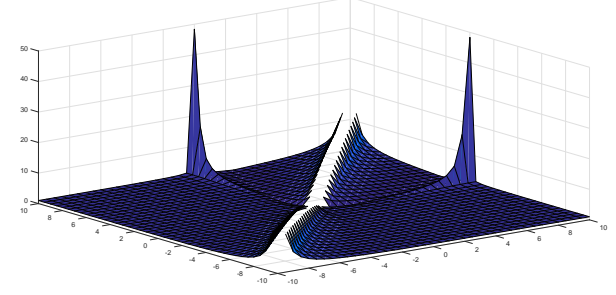

(c) $\gamma=0.5$

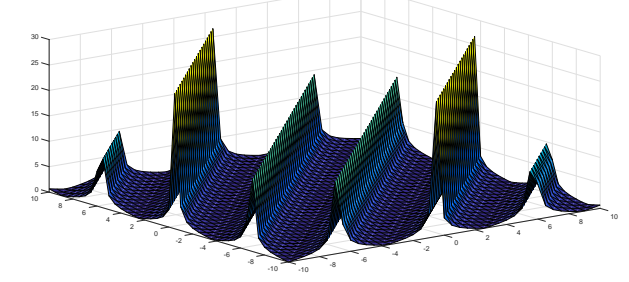

(b) $\gamma=1$

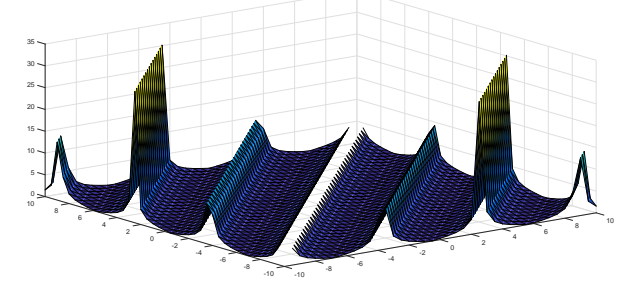

(d) $\gamma=1$

Figure 3: Solitary wave profile of $u_{1,2}$ and $u_{3,4}$ appear in Eq.17 


\subsection{Exact solutions for space-time FEW equation}

Consider the conformable space-time fractional EW Equation as follows [43,44].

$$
D_{t}^{\gamma} u(x, t)+\epsilon D_{x}^{\gamma} u^{2}(x, t)-\delta D_{x x t}^{3 \gamma} u(x, t)=0, \quad t>0 \quad 0<\gamma \leq 1,
$$

Taking the transformation (3) as $\xi=k \frac{x^{\gamma}}{\gamma}-l \frac{t^{\gamma}}{\gamma}$, and integrating w.r.t. $\xi$, we get

$$
\delta l k^{2} U^{\prime \prime}-l U+\epsilon k U^{2}=0 .
$$

Through balancing the terms $U^{\prime \prime}$ and $U^{2}$, it is easy to select $N=2$, and the non-trivial solution (5(a)) becomes

$$
U(\xi)=A_{1} \cosh (\omega)+B_{1} \sinh (\omega)+A_{0}+A_{2} \sinh (\omega) \cosh (\omega)+B_{2} \sinh ^{2}(\omega) .
$$

Case-1: $\frac{d \omega}{d \xi}=\sinh (\omega)$ By setting the above non-trivial solution in reduced equation Eq.(25) and equating the coefficients to zero in the resultant equation, a set of nonlinear algebraic equations is obtained and whose solution yields the following new exact solutions to conformable space-time FEW equation that can be written as

$$
\begin{gathered}
\text { Case }-1.1: A_{0}=0, A_{1}=0, A_{2}= \pm \frac{3 \sqrt{\delta} l}{\epsilon}, B_{1}=0, B_{2}=\mp \frac{3 \sqrt{\delta} l}{\epsilon}, k= \pm \frac{1}{\sqrt{\delta}} \\
u_{1,2}(x, t)= \pm \frac{3 \sqrt{\delta} l}{2 \epsilon} \operatorname{sech}^{2}\left(\frac{k \frac{x^{\beta}}{\beta}-l \frac{t^{\gamma}}{\gamma}}{2}\right) \\
A_{0}=0, A_{1}=0, A_{2}=\mp \frac{3 \sqrt{\delta} l}{\epsilon}, B_{1}=0, B_{2}=\mp \frac{3 \sqrt{\delta} l}{\epsilon}, k= \pm \frac{1}{\sqrt{\delta}} \\
u_{3,4}(x, t)=\mp \frac{3 \sqrt{\delta} l}{2 \epsilon} \operatorname{csch}^{2}\left(\frac{k \frac{x^{\beta}}{\beta}-l \frac{t^{\gamma}}{\gamma}}{2}\right) \\
\text { Case- } 1.2: A_{0}=\frac{i \sqrt{\delta} l}{\epsilon}, A_{1}=0, A_{2}=\mp \frac{3 i \sqrt{\delta} l}{\epsilon}, B_{1}=0, B_{2}=\frac{3 i \sqrt{\delta} l}{\epsilon}, k=\frac{-i}{\sqrt{\delta}} \\
u_{5}(x, t)=1-\frac{3 i \sqrt{\delta} l}{2 \epsilon} \operatorname{sech}^{2}\left(\frac{k \frac{x^{\beta}}{\beta}-l \frac{t^{\gamma}}{\gamma}}{2}\right) \\
u_{6}(x, t)=1+\frac{3 i \sqrt{\delta} l}{2 \epsilon} \operatorname{csch}^{2}\left(\frac{k \frac{x^{\beta}}{\beta}-l \frac{t^{\gamma}}{\gamma}}{2}\right)
\end{gathered}
$$




$$
\begin{gathered}
A_{0}=-\frac{i \sqrt{\delta} l}{\epsilon}, A_{1}=0, A_{2}=\mp \frac{3 i \sqrt{\delta} l}{\epsilon}, B_{1}=0, B_{2}=-\frac{3 i \sqrt{\delta} l}{\epsilon}, k=\frac{i}{\sqrt{\delta}} \\
u_{7}(x, t)=-\frac{i \sqrt{\delta} l}{2 \epsilon}\left(\cosh \left(k \frac{x^{\beta}}{\beta}-l \frac{t^{\gamma}}{\gamma}\right)+2\right) \operatorname{csch}^{2}\left(\frac{k \frac{x^{\beta}}{\beta}-l \frac{t^{\gamma}}{\gamma}}{2}\right) \\
u_{8}(x, t)=-\frac{i \sqrt{\delta} l}{2 \epsilon}\left(\cosh \left(k \frac{x^{\beta}}{\beta}-l \frac{t^{\gamma}}{\gamma}\right)-2\right) \operatorname{sech}^{2}\left(\frac{k \frac{x^{\beta}}{\beta}-l \frac{t^{\gamma}}{\gamma}}{2}\right) \\
\text { Case-1.3: } A_{0}=0, A_{1}=0, A_{2}=0, B_{1}=0, B_{2}=\mp \frac{3 \sqrt{\delta} l}{\epsilon}, k= \pm \frac{1}{2 \sqrt{\delta}} \\
u_{9,10}(x, t)=\mp \frac{3 \sqrt{\delta} l}{\epsilon} \operatorname{csch}^{2}\left(k \frac{x^{\beta}}{\beta}-l \frac{t^{\gamma}}{\gamma}\right) \\
A_{0}=\mp \frac{2 i \sqrt{\delta} l}{\epsilon}, A_{1}=0, A_{2}=0, B_{1}=0, B_{2}=\mp \frac{3 i \sqrt{\delta} l}{\epsilon}, k= \pm \frac{i}{2 \sqrt{\delta}} \\
u_{11,12}(x, t)=\mp \frac{i \sqrt{\delta} l}{\epsilon}\left(3 \operatorname{csch}^{2}\left(k \frac{x^{\beta}}{\beta}-l \frac{t^{\gamma}}{\gamma}\right)+2\right)
\end{gathered}
$$

Case-2: $\frac{d \omega}{d \xi}=\cosh (\omega)$ and for $N=2$, a set of nonlinear equations is obtained as which its solution yields the following new exact solutions to conformable space-time FEW equation that can be written as

$$
\begin{aligned}
& \text { Case }-2.1: A_{0}=\mp \frac{\sqrt{\delta} l}{\epsilon}, A_{1}=0, A_{2}=0, B_{1}=0, B_{2}=\mp \frac{3 \sqrt{\delta} l}{\epsilon}, k= \pm \frac{1}{2 \sqrt{\delta}} \\
& u_{1,2}(x, t)=\mp \frac{\sqrt{\delta} l}{\epsilon}\left(3 \cot ^{2}\left(k \frac{x^{\beta}}{\beta}-l \frac{t^{\gamma}}{\gamma}\right)+1\right) \\
& A_{0}=\mp \frac{3 i \sqrt{\delta} l}{\epsilon}, A_{1}=0, A_{2}=0, B_{1}=0, B_{2}=\mp \frac{3 i \sqrt{\delta} l}{\epsilon}, k= \pm \frac{i}{2 \sqrt{\delta}} \\
& u_{3,4}(x, t)=\mp \frac{3 i \sqrt{\delta} l}{\epsilon} \csc ^{2}\left(k \frac{x^{\beta}}{\beta}-l \frac{t^{\gamma}}{\gamma}\right) \\
& \text { Case }-2.2: A_{0}=\mp \frac{2 \sqrt{\delta} l}{\epsilon}, A_{1}=0, A_{2}=\mp \frac{3 \sqrt{\delta} l}{\epsilon}, B_{1}=0, B_{2}=\mp \frac{3 \sqrt{\delta} l}{\epsilon}, k= \pm \frac{1}{\sqrt{\delta}} \\
& u_{5,6}(x, t)= \pm \frac{\sqrt{\delta} l}{2 \epsilon}\left(\cos \left(k \frac{x^{\gamma}}{\gamma}-l \frac{t^{\gamma}}{\gamma}\right)-2\right) \sec ^{2}\left(\frac{k \frac{x^{\gamma}}{\gamma}-l \frac{t^{\gamma}}{\gamma}}{2}\right) \\
& A_{0}=\mp \frac{2 \sqrt{\delta} l}{\epsilon}, A_{1}=0, A_{2}= \pm \frac{3 \sqrt{\delta} l}{\epsilon}, B_{1}=0, B_{2}=\mp \frac{3 \sqrt{\delta} l}{\epsilon}, k= \pm \frac{1}{\sqrt{\delta}}, \\
& u_{7,8}(x, t)=\mp \frac{\sqrt{\delta} l}{2 \epsilon}\left(\cos \left(k \frac{x^{\gamma}}{\gamma}-l \frac{t^{\gamma}}{\gamma}\right)+2\right) \csc ^{2}\left(\frac{k \frac{x^{\gamma}}{\gamma}-l \frac{t^{\gamma}}{\gamma}}{2}\right)
\end{aligned}
$$




$$
\begin{aligned}
\text { Case }-2.3: A_{0} & =\mp \frac{3 i \sqrt{\delta} l}{\epsilon}, A_{1}=0, A_{2}=\mp \frac{3 i \sqrt{\delta} l}{\epsilon}, B_{1}=0, B_{2}=\mp \frac{3 i \sqrt{\delta} l}{\epsilon}, k= \pm \frac{i}{\sqrt{\delta}} \\
u_{9,10}(x, t) & =\mp \frac{3 i \sqrt{\delta} l}{2 \epsilon} \sec ^{2}\left(\frac{k \frac{x^{\gamma}}{\gamma}-l \frac{t^{\gamma}}{\gamma}}{2}\right) \\
A_{0} & =\mp \frac{3 i \sqrt{\delta} l}{\epsilon}, A_{1}=0, A_{2}= \pm \frac{3 i \sqrt{\delta} l}{\epsilon}, B_{1}=0, B_{2}=\mp \frac{3 i \sqrt{\delta} l}{\epsilon}, k= \pm \frac{i}{\sqrt{\delta}} \\
u_{11,12}(x, t) & =\mp \frac{3 i \sqrt{\delta} l}{2 \epsilon} \csc ^{2}\left(\frac{k \frac{x^{\gamma}}{\gamma}-l \frac{t^{\gamma}}{\gamma}}{2}\right)
\end{aligned}
$$

Remark. If $u(x, t)=v(x, t)+1$ and $\epsilon=\frac{1}{2}$, then the space-time fractional EWE becomes space-time fractional BBM equation [45]. Therefore, we can easily obtain the explicit exact solution of the said equation with the help of given solutions of space-time fractional EWE.

\subsection{Numerical simulation for solutions of space-time FEW equation}

The numerical simulation for solutions of Eq.(24) is given here for different $\gamma$-values corresponding to $\epsilon=1=l$, and $\delta=2$.

Case-I: The two solutions given in Eq.(29) of Eq.(24) are graphed in figures 4(a)-4(d) and $5(\mathrm{a})-5(\mathrm{~d})$ for $\gamma=0.5,1$ and $t=0$ respectively.

Case-II: The graphs $6(\mathrm{a})-6(\mathrm{~d})$ and $7(\mathrm{a})-7(\mathrm{~d})$ reveal the two periodic wave solutions given in Eq.(34) of Eq.(24) for $\gamma=0.5, \gamma=1$ and $t=0$ respectively. 


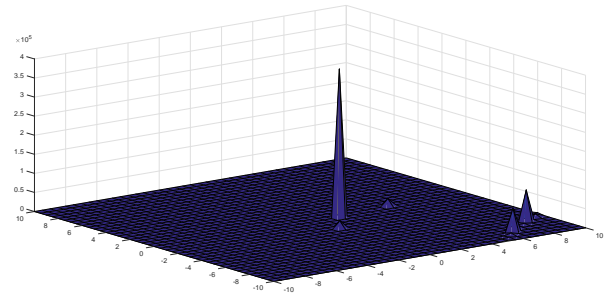

(a) $\gamma=0.5$

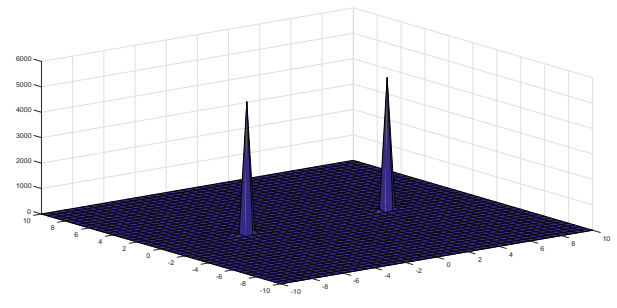

(c) $\gamma=1$

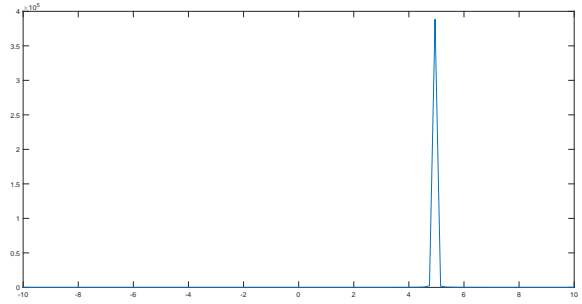

(b) $\gamma=0.5 \& t=0$

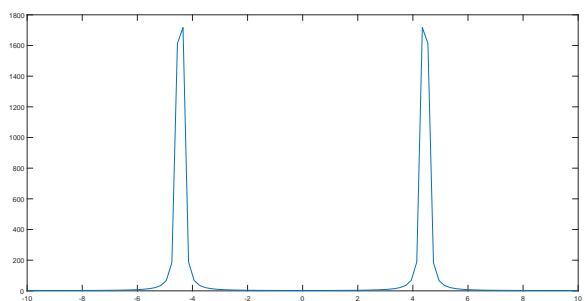

(d) $\gamma=1 \& t=0$

Figure 4: Solitary wave profiles of $u_{5}$ appears in Eq.29

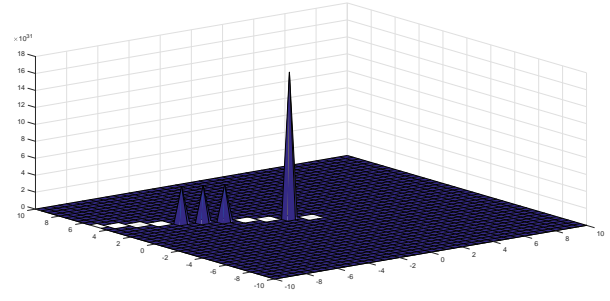

(a) $\gamma=0.5$

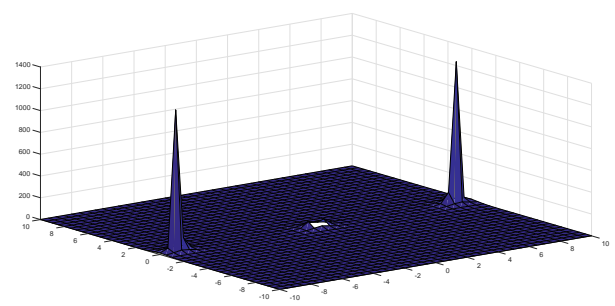

(c) $\gamma=1$

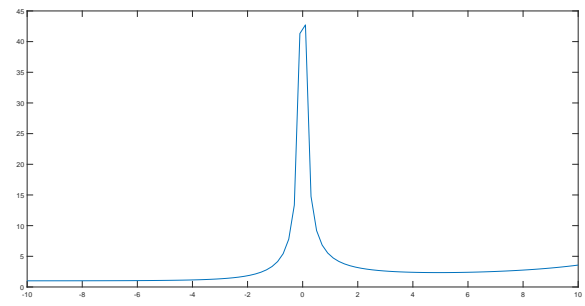

(b) $\gamma=0.5 \& t=0$

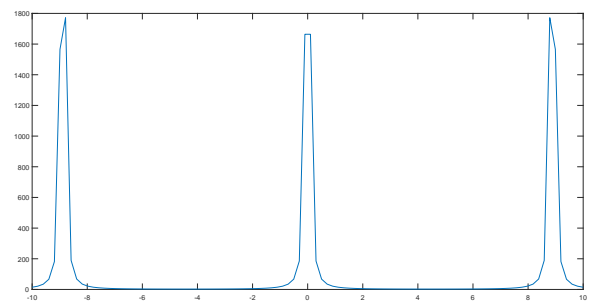

(d) $\gamma=1 \& t=0$

Figure 5: Solitary wave profile of $u_{6}$ appears in Eq.29 


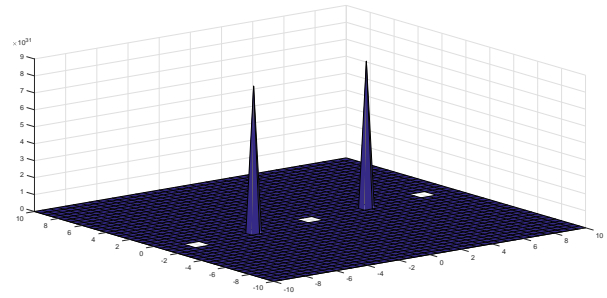

(a) $\gamma=0.5$

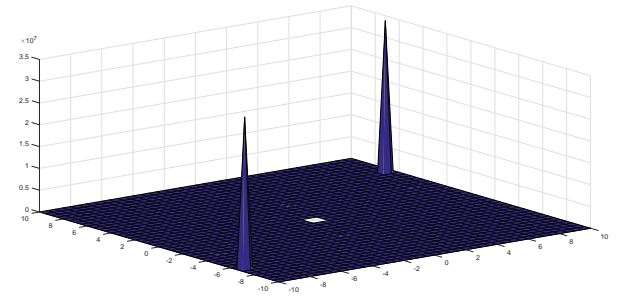

(c) $\gamma=1$

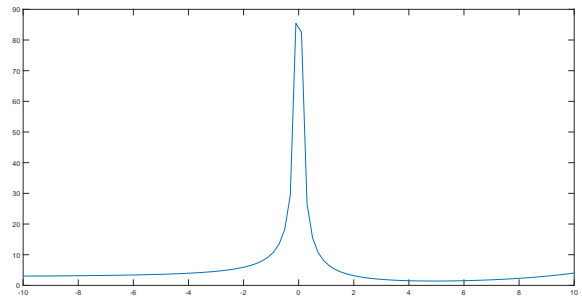

(b) $\gamma=0.5 \& t=0$

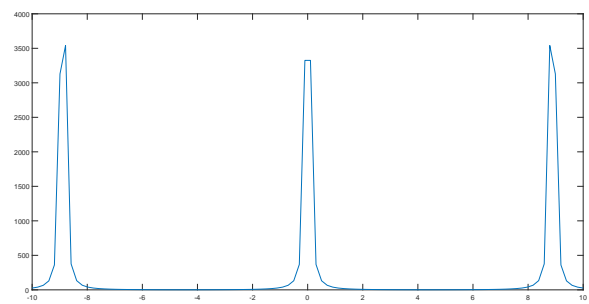

(d) $\gamma=1 \& t=0$

Figure 6: Solitary wave profile of $u_{1,2}$ appear in Eq.34

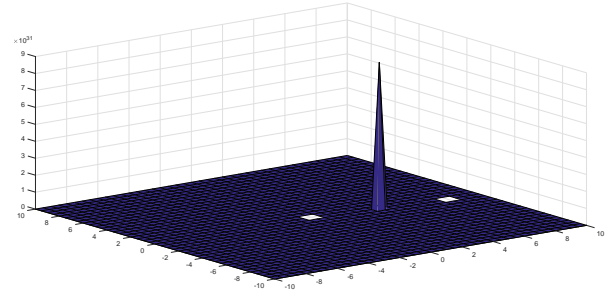

(a) $\gamma=0.5$

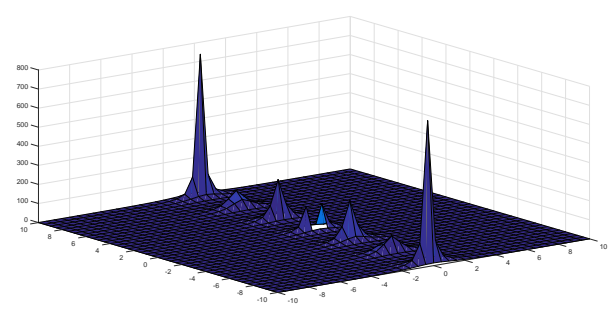

(c) $\gamma=1$

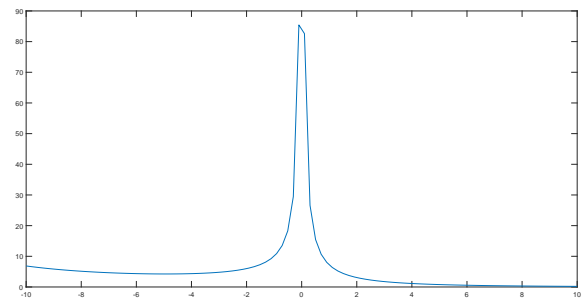

(b) $\gamma=0.5 \& t=0$

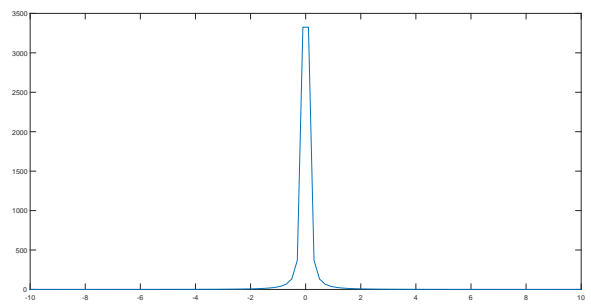

(d) $\gamma=1 \& t=0$

Figure 7: Solitary wave profile of $u_{3,4}$ appears in Eq.34 


\section{Conclusion}

The study explored a wide range of new explicit and exact soliton type solutions for conformable space-time fractional EW and modified EW equations via hyperbolic function approach. The solutions are constructed in various forms including hyperbolic and trigonometric functions or both. Many of the solutions are of the multi wave solutions due to its functional structure as some solutions are of the complex wave forms. These solutions are verified by inserting back in the original equations with the aid of symbolic computation in Mathematica. Furthermore, the numerical simulation of some solutions has been left for the reader to visualize them.

\section{References}

[1] Igor Podlubny. Fractional differential equations: an introduction to fractional derivatives, fractional differential equations, to methods of their solution and some of their applications. Mathematics in Science and Engineering, 198, 1999.

[2] Guo Boling, $\mathrm{Pu}$ Xueke, and Huang Fenghui. Fractional partial differential equations and their numerical solutions. World Scientific, 2015.

[3] G. Samko, A.A. Kilbas, and Marichev. Fractional Integrals and Derivatives: Theory and Applications. Gordon and Breach, Yverdon, 1993.

[4] A. Kilbas, M. H. Srivastava, and J. J. Trujillo. Theory and application of fractional differential equations. North Holland Mathematics Studies, 204, 2006.

[5] Kenneth S Miller and Bertram Ross. An introduction to the fractional calculus and fractional differential equations. 1993.

[6] Guy Jumarie. Modified riemann-liouville derivative and fractional taylor series of nondifferentiable functions further results. Computers 8 Mathematics with Applications, 51(9-10):1367-1376, 2006.

[7] Michele Caputo and Mauro Fabrizio. A new definition of fractional derivative without singular kernel. Progress in fractional differentiation and applications, 1(2):73 - 85, 2015 .

[8] Zheng-Biao Li. An extended fractional complex transform. International Journal of Nonlinear Sciences and Numerical Simulation, 11(Supplement):335-338, 2010.

[9] R. Khalil, M. Al Horani, A. Yousef, and M. Sababheh. A new defination of fractional derivative. J.Comput.Appl.Math., 264:65-70, 2014. 
[10] Ji-Huan He, S.K. Elagan, and Z.B. Li. Geometrical explanation of the fractional complex transform and derivative chain rule for fractional calculus. Physics Letters A, 376(4):257 - 259, 2012.

[11] Zheng-Biao Li and Ji-Huan He. Fractional complex transform for fractional differential equations. Mathematical and Computational Applications, 15(5):970-973, 2010.

[12] D Howell Peregrine. Long waves on a beach. Journal of fluid mechanics, 27(4):815-827, 1967.

[13] Fan HL. The classiication of single trawling wave solutions to the generalized equalwidth equation. Appl. Math. Comput., 219(2):748-754, 2012.

[14] Cary JR. Morrison PJ, Meiss JD. Scattering of regularized-long-wave solitry waves. Physica D: Nonlinear Phenomena, 11(3):324-336, 1984.

[15] K. R. Raslan. Collocation method using quartic b-spline for the equal width (ew) equation. Applied Mathematics and Computation, 168(2):795-805, 2005.

[16] L.R.T. Gardner, G. A. Gardner, F. A. Ayoub, and N.K. Amein. Simulations of the ew undular bore. Communications in Numerical Methods in Engineering, 13(7):583-592, 1997.

[17] S. I. Zaki. A least-squares finite element scheme for the ew equation. Computer Methods in Applied Mechanics and Engineering, 189(2):587-594, 2000.

[18] S. I. Zaki. Solitary waves induced by the boundary forced ew equation. Computer methods in applied mechanics and engineering, 190(37-38):4881-4887, 2001.

[19] Hossein Aminikhah, Amir HosseinRefahi Sheikhani, and HAdi Rezazadeh. Subequation method for the fractional regularized long-wave equations with conformable fractional derivatives. ScientiaIranica. Transaction B, Mechanical Engineering, 23(3):1048, 2016.

[20] H. Rezazadeh. New solitons solutions of the complex ginzburg-landau equation with kerr law nonlinearity. Optik, 167:218-227, 2018.

[21] B. Lu. The first integral method for some time fractional differential equations. $J$. Math. Anal. Appl., 395:684-693, 2012.

[22] M. Eslami. Trial solution technique to chiral nonlinear schrodinger's equation in $(1+2)-$ dimensions. Nonlinear Dynam, 85(2):813-816, 2016.

[23] M. Younis and Asim Zafar. Exact solutions to nonlinear differential equations of fractional order via $\left(\frac{G^{\prime}}{G}\right)$-expansion method. Appl. Math, 5:1-6, 2014. 
[24] H. Aminikhah, A.H Refahi Sheikhani, and H. Rezazadeh. Travelling wave solutions of nonlinear systems of pdes by using the functional variable method. Boletim da Sociedade Paranaense de Matemática, 34.

[25] A.Korkmaz. Complex wave solutions to mathematical biology models i: Newellwhitehead-segel and zeldovich equations. Journal of Computational and Nonlinear Dynamics, 13(8):1-7, 2018.

[26] H. Rezazadeh, A. Korkmaz, M. Eslami, J. Vahidi, and R. Asghari. Traveling wave solution of conformable fractional generalized reaction duffing model by generalized projective riccati equation method. Optical and Quantum Electronics, 50(3):150, 2018.

[27] M.S. Islam, M.B. Hossain, and M.A. Salam. Soliton-like solutions for some nonlinear evolution equations through the generalized kudryashov method. Global Journal of Science Frontier Research: F Mathematics and Decision Sciences, 17(7):1-15, 2007.

[28] F. Mahmud, M. Samsuzzoha, and M.A. Akbar. The generalized kudryashov method to obtain exact traveling wave solutions of the phi-four equation and the fisher equation. Results in Physics, 7:4296-4302, 2017.

[29] K. Hosseini, E. Yazdani Bejarbaneh, A. Bekir, and M. Kaplan. New exact solutions of some nonlinear evolution equations of pseudoparabolic type. Optical and Quantum Electronics, 49(7):241, 2017.

[30] Z. Ayati, K. Hosseini, and M. Mirzazadeh. Application of kudryashov and functional variable methods to the strain wave equation in microstructured solids. Nonlinear Engineering, 6:25-29, 2017.

[31] A. Korkmaz. Exact solutions to $(3+1)$ conformable time fractional jimbo-miwa, zakharov-kuznetsov and modified zakharov-kuznetsov equations. Communications in Theoretical Physics, 67(5):479, 2017.

[32] K. Hosseini, F. Samadani, D. Kumar, and M. Faridi. New optical solitons of cubicquartic nonlinear schrödinger equation. Optik, 157:1101 - 1105, 2018.

[33] Alper Korkmaz. Explicit exact solutions to some one dimensional conformable time fractional equations. Waves in Random and Complex Media, 29(1):124-137, 2019.

[34] E. Fan. Extended tanh-function method and its application to nonlinear equation. Phys. Lett. A., 277:212-218, 2000.

[35] AM. Wazwaz. The extended tanh method for the zakharov-kuznestsov(zk) equation, the modified zk equation, and its generalized forms. Commun. Nonlinear Sci., 13:10391047, 2008. 
[36] Kamyar Hosseini, Jalil Manafian, Farzan Samadani, Mohammadreza Foroutan, Mohammad Mirzazadeh, and Qin Zhou. Resonant optical solitons with perturbation terms and fractional temporal evolution using improved $\tan (\phi(\eta) / 2)$-expansion method and exp function approach. Optik, 158:933 - 939, 2018.

[37] A. T. Ali and E. R. Hassan. General $\exp _{a}$ function method for nonlinear evolution equations. Appl. Math. Comput., 217:451-459, 2010.

[38] K. Hosseini, Z. Ayati, and R. Ansari. New exact solution of the tzitzéica type equations in nonlinear optics using the $\exp _{a}$ function method. J. Mod. Opt., 65(7):847-851, 2018.

[39] K. Hosseini, A. Zabihi, F. Samadani, and R. Ansari. New explicit exact solutions of the unstable nonlinear schrödinger's equation using the $\exp _{a}$ and hyperbolic function methods. Optical and Quantum Electronics, 50(2):82, 2018.

[40] E. M. E. Zayed and A. G. Al-Nowehy. Generalized kudryashov method and general $\exp _{a}$ function method for solving a high order nonlinear schrödinger equation. $J$. Space Explor., 6:1-26, 2017.

[41] Fuding Xie, Zhenya Yan, and Hongqing Zhang. Explicit and exact traveling wave solutions of whitham-broer-kaup shallow water equations. Physics Letters A, 285(1):76 $-80,2001$.

[42] Chenglin Bai. Exact solutions for nonlinear partial differential equation: a new approach. Physics Letters A, 288(3):191 - 195, 2001.

[43] Alper Korkmaz. Exact solutions of space-time fractional ew and modified ew equations. Chaos, Solitons and Fractals, 96:132-138, 2017.

[44] K Hosseini and Z Ayati. Exact solutions of space-time fractional ew and modified ew equations using kudryashov method. Nonlinear Science Letters A Mathematics, Physics and Mechanics, 7(2):58-66, 2016.

[45] Hossam A Ghany and Ashraf Fathallah. White-noise functional solutions for wick-type stochastic time-fractional benjamin-bona-mahony equation. International Journal of Differential Equations and Applications, 13(1), 2014. 